\title{
Modeling method of combat mission based on OODA loop
}

\author{
Xiao Li, and Desheng Liu* \\ Science and Technology on Complex Electronic System Simulation Laboratory, Space Engineering \\ University, Beijing, China
}

\begin{abstract}
Today, modern warfare has shifted from weapon-centric operations to network-centric system operations, which has led to a linear increase in the complexity of combat missions. When a commander faces a high-level mission, how to model the entire combat mission is a critical step, and it is also the basis for the generation of subsequent combat plans and combat command and control. Aiming at this problem, this paper proposes a task modeling method based on the OODA loop. This method first decomposes the mission task, and defines four meta tasks based on the OODA loop theory, and finally analyzes the meta tasks from the perspective of time and information. The mission relationship is defined, which can realize the modeling and formal description of the entire combat mission process, and provide support for the follow-up combat links.
\end{abstract}

Keywords: Mission modelling, Mission breakdown, OODA, Information flow modeling.

\section{Introduction}

Under the conditions of systematic combat, the complexity of combat missions has gradually increased, which has brought various difficulties to combat modeling. This complexity is embodied in the following aspects: First, the missions assigned by the highlevel are generally vague, cannot be understood by the combat unit, and are not conducive to the commander's decision-making in the face of massive combat resources. Second, in the course of combat, many different tasks are related to each other, and there are many kinds of coordination relationships between different types of tasks, which causes the combat unit to not be smooth when completing tasks. Finally, the indicators of combat missions are becoming more abundant, and there are various scenarios that can complete the mission, which will have an impact on the decision-making of combat commanders.

In response to this problem, the article[1] proposes a method of task decomposition and combat task modeling, which can realize the decomposition of fuzzy mission tasks and carry out a certain formal description to establish the relationship between task requirements and weapon equipment capabilities. Aiming at the optimization problem of

*Corresponding author: liudsnudt@126.com 
task decomposition in cooperative operations, the article[2] constructs a quantitative model of task coordination correlation, defines several basic concepts in combat tasks, and analyzes the coordination relationship between tasks from the three dimensions of time, logic, and function. Formal description. Based on the structural decomposition of maritime area air defense combat missions, the article[3] establishes a DoDAF-based area defense combat activity model view and combat dynamic description view. On the basis of the above methods, this paper defines different types of meta tasks after task decomposition based on the OODA ring theory, and constructs a variety of relationships between meta tasks from the perspective of time and information to achieve multi-dimensional and multidimensional Combat mission modeling can formally describe the entire combat process in more detail.

\section{Mission breakdown}

As mentioned above, high-level missions are usually vague and general, and difficult to be understood by combat platforms, making it difficult for combat commanders to plan combat missions and generate combat plans. Therefore, the task decomposition technique should be applied first, which divides the complex tasks that cannot be performed directly into meta tasks that can be performed directly, so as to reduce the complexity of decision making[4].

(1) Mission $M$ : A mission assignment is an assignment given to a level or region commander, usually as an indication of superior intent. Missions are macroscopic, general and even vague, and cannot be understood by combat units and command systems. Therefore, the execution process needs to be decomposed and explained in detail.

(2) Sub task: A subtask is a task that is decomposed by the upper-level task and can be further decomposed. If it cannot be decomposed, it can be transformed into a meta task.

(3) Meta task $T$ :meta task is the most basic tasks that can be understood and executed directly and can no longer be decomposed.

(4) Task decomposition: According to certain principles or methods, the process of subdividing more complex tasks into meta tasks that can be understood and directly executed by operational resources is also the process of transforming a macro mission into a collection of several meta tasks.

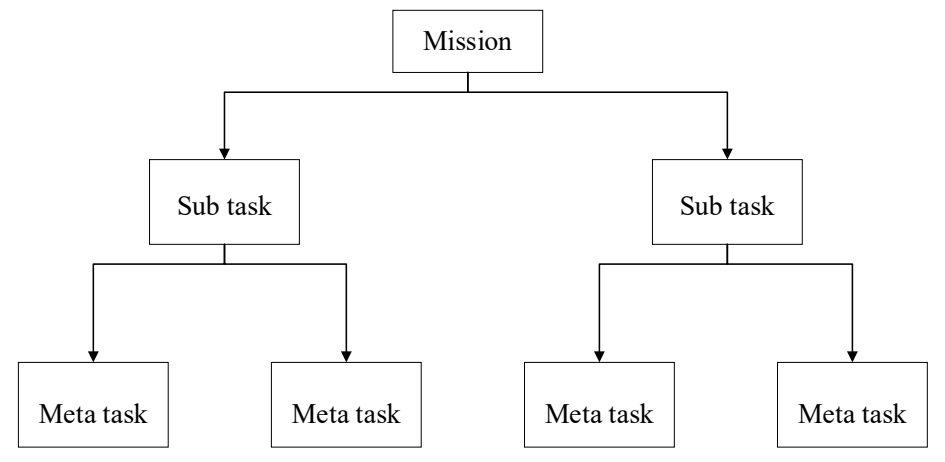

Fig. 1. Mission breakdown.

Task decomposition refines an abstract complex task into a more specific and relatively independent meta task, which is an optimization decision process with granularity from coarse to fine. The smaller the granularity, the finer the task, the richer the easy-tounderstand information, and the more stringent the constraints. And decomposition of a mission to multiple sub tasks, and each task can also be broken down into sub tasks more 
again, so every time after decomposition results, are decomposed granularity of judgment, is the judgment after decomposition of each child task decomposition, whether to need to continue if need be, decomposition, continue if unable to decomposition, The sub task can be treated as a meta task.

Through the above process, mission tasks can be transformed into collections of meta tasks:

$M=\left\{T_{1}, T_{2}, T_{3}, T_{4}, \ldots, T_{n}\right\} \cdot(n$ is the number of meta tasks $)$

\section{Meta task modeling}

In the previous section, the mission task has been decomposed into a metatask collection $M=\left\{T_{1}, T_{2}, T_{3}, T_{4}, \ldots, T_{n}\right\}$, in order to better describe the process of achieving mission tasks $M=\left\{T_{1}, T_{2}, T_{3}, T_{4}, \ldots, T_{n}\right\}$, each meta task in the collection, can enable the weapon equipment system to better understand and perform a range of operational tasks.

\subsection{OODA operational theory}

For systematic operations, the OODA theory[5] proposed by John Boyd, an American Air Force major, can clearly describe the process of the entire system and carry out a high degree of abstraction. In other words, a closed loop of "Observe--Orient--Decide--Act" is shown in the figure 2.

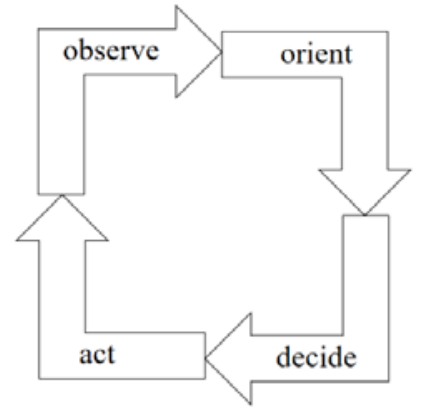

Fig. 2. The OODA theory.

In Boyd's theory, systematic operations can be described as follows: firstly, reconnaissance and early warning equipment is used to detect the combat space to find suspicious targets, and sensor equipment is used to collect intelligence of suspicious targets, including early warning information, target location information and target status information, etc. Then processing and analyzing the collected intelligence information, judging its accuracy, target identification and battlefield situation judgment; According to the results obtained above, we should make operational decisions, formulate operational plans and issue operational instructions based on the existing conditions; Finally, according to the decision results and issued instructions, according to the battle plan to take the corresponding action.

\subsection{Meta task modeling based on OODA theory}

The four processes in OODA combat theory describe the process of systematic operations, that is, the four processes of completing missions and tasks. Therefore, the meta task set obtained after mission tasks are decomposed must also be included in these four processes. 
Therefore, based on OODA operational theory, meta tasks are divided into four types in this study according to the four basic processes in the theory: reconnaissance and early warning task, intelligence processing task, decision control task and response execution task, as shown in the figure 3 .

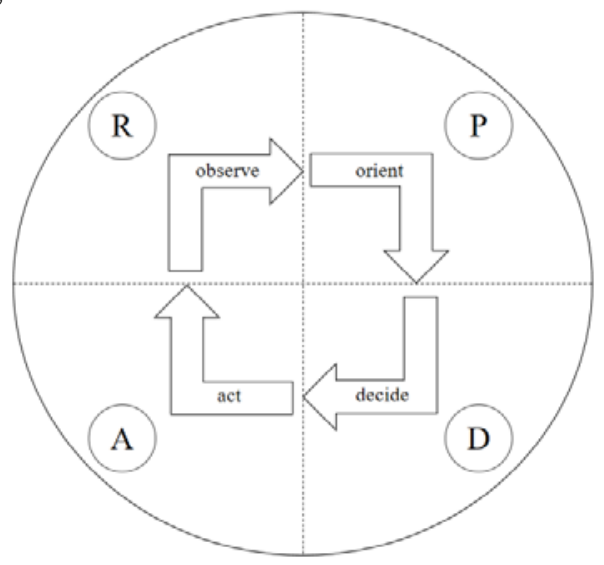

Fig. 3. Meta task modeling.

Definition 3.1 Reconnaissance and early warning mission (Reconnaissance, $R$ ):

The task of detection and reconnaissance of various targets in battlefield space is generally realized by carrying various types of sensors such as radar, photoelectric and signal on various reconnaissance and early warning platforms such as sea, land, air, sky and underwater. Mission targets include a variety of targets in the battlefield space, including local military bases, airports, camps and other forces, as well as enemy aircraft, ships, cruise missiles and other equipment. Can be described as:

$$
R=\{\text { number, location, range, datatype, accuracy, demand }\}
$$

(1) Number: Task number, which is used to identify the task.

(2) Location: The locations of reconnaissance and early warning missions may be represented by geographical coordinates.

(3) Range: The detection radius of reconnaissance and early warning missions can determine the scope of the detection area by the detection radius and location.

(4) Datatype: It specifies the data types of intelligence information to be collected by reconnaissance and early warning missions, such as sound, signals and images.

(5) Accuracy: The accuracy of intelligence information received by reconnaissance and early warning missions is required to be measured by a number $(0,1)$, where 1 represents complete consistency and 0 represents complete inconsistency.

(6) Demand: The requirement of a task for weapon equipment capability can be expressed as a requirement capability vector.

Definition 3.2 Information processing task (Processor, $P$ ): It is a functional entity that performs related processing of target intelligence in battlefield space, including the fusion, feature extraction and recognition of multi-source intelligence data, so as to form real-time continuous, clear and accurate intelligence, which can be used for subsequent decision-making and task execution. Can be described as:

$$
P=\{\text { number, location, range, datatypes, amount, time, demand }\}
$$

(1) Number: Same as 3.1 
(2) Location: The location of an intelligence processing task can be represented by geographical coordinates.

(3) Range: The jurisdiction of an intelligence processing task. The intelligence source of an intelligence processing task can be determined by jurisdiction and location.

(4) Datatypes: The type of information to be processed by an intelligence processing task. It is generally a collection of multiple types.

(5) Amount: The amount of data to be processed by an intelligence processing task.

(6) Time: The estimated time for an intelligence processing task to process complete data. The data processing capability is determined by determining the amount of data and the processing time.

(7) Demand: Same as 3.1

Definition 3.3 Decision control task (Decision, $D$ ): Analyze and judge the battlefield space situation, provide combat plans (including specific decision-making content such as when and where to use what combat resources and how to achieve what objectives), and conduct real-time command and control of troops and weapons and equipment platforms in their own combat resources. Can be described as:

$$
P=\{\text { number, location, range, amount, time, level, demand }\}
$$

(1) Number: Same as 3.1

(2) Location: The location of the decision control task can be represented by geographical coordinates.

(3) Range: Decisions control the scope of the mission. By jurisdiction and location, the scope of operational resources that can be controlled can be determined.

(4) Amount: Decisions control how much data a task will process.

(5) Time: The ability required by the decision control task can be determined by the estimated time and total data.

(6) Level: The operational level of the control decision task is represented by a number from 1 to 3, with 1 being strategic, 2 campaign, and 3 tactical.

(7) Demand: Same as 3.1

Definition 3.9 Response execution task (Action, $A$ ): After receiving the combat command or combat plan, carry out combat operations according to its content and complete the expected objectives. Can be described as:

$$
A=\{\text { number, location, range, demand }\}
$$

(1) Number: Same as 3.1

(2) Location: The location of the response to perform the task can be represented by geographic coordinates.

(3) Range: Response to the scope of the implementation of the task, through the scope of activities and locations to determine the response to the implementation of the area.

(4) Demand: Same as 3.1.

\section{Meta task relationship modeling}

After the meta task modeling in the previous section, the formal description of each meta task is realized, and the goal of each type of meta task is specified. The relationship construction of meta tasks can describe all the elements in the meta task set. The interrelationship of tasks clarifies how meta tasks cooperate with each other to achieve the overall mission task. Under system combat conditions, the relationship between meta tasks 
is intricate and complex. This article formally defines the relationship between meta tasks from the perspectives of time and information.

\subsection{Meta task time flow relationship modeling}

In actual combat scenarios, the execution of meta tasks must follow a certain order in time, so the time relationship between meta tasks can be constructed. This article [6] uses the time relationship studied in the literature to model the time of meta tasks.

(1) Serial meets relationship: If the meta tasks $T_{i}, T_{j}$ are carried out in chronological order without interval, then it is called the serial meets relationship, which is recorded as Serial $_{\text {meets }}\left(T_{i}, T_{j}\right)$.

(2) Serial before relationship: If the meta tasks $T_{i}, T_{j}$ are performed after an interval of seconds in time sequence, it is called the serial before relationship, which is recorded as Serial $_{\text {before }}\left(T_{i}, T_{j}\right)^{\cdot}$

(3) Serial overlaps relationship: If the meta tasks are carried out sequentially in chronological order, and there is overlap in them, it is called the serial overlaps relationship, which is recorded as Serial overlaps $_{i}\left(T_{i}, T_{j}\right)$.

(4) Parallel during relationship: If the meta tasks $T_{i}, T_{j}$ are carried out in the same time period, and the start and end time are the same, then it is called a parallel inclusion relationship, and it is recorded as

$$
\text { parallel }_{\text {during }}\left(T_{i}, T_{j}\right) \text {. }
$$

(5) Parallel stars relationship: If the meta tasks $T_{i}{ }^{\prime} T_{j}$ are performed in the same time period and the start time is the same, then it is called the parallel starts relationship, which is recorded as parallel $_{\text {starts }}\left(T_{i}, T_{j}\right)$.

(6) Parallel finishes relationship: If the meta task $T_{i}, T_{j}$ are performed in the same time period and the finish time is the same, then it is called the parallel finishes relationship, which is recorded as

$$
\text { parallel }_{\text {finishes }}\left(T_{i}, T_{j}\right) \text {. }
$$

(7) Parallel equal relationship: If the meta tasks $T_{i}{ }^{\prime} T_{j}$ are carried out in the same time period and the end time is the same, then it is called the parallel equal relationship, which is recorded as

$$
\text { parallel }_{\text {equal }}\left(T_{i}, T_{j}\right) \text {. }
$$

The graphic explanation is shown in Figure 4.

\subsection{Meta task information flow relationship modeling}

After the form of war has entered the information age, with the support of information network technology, the combat space can include six dimensions of land, sea, air, space, and the Internet.

During combat, the span of information exchange is large and the interaction is frequent. Tasks can be completed in real time, accurately, flexibly and efficiently, and meta task relationship modeling is also required for information flow. The purpose is to analyze how information flows between tasks and achieve the mission collaboratively. 

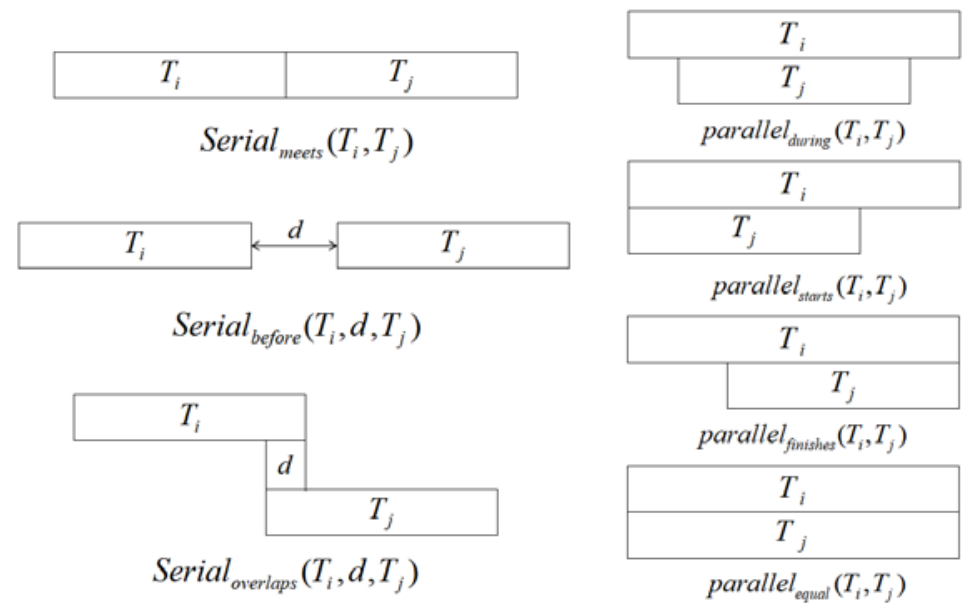

Fig. 4. Meta task relationship modelling.

Relation modeling for information flow requires different types of meta tasks to build different relationships. In the previous section, meta task modeling divides meta tasks into four types, including 16 types of relationships, and removes two different types. Common and low-probability relationships, a total of 13 meta task information flow relationships are defined, as shown in the table.

Table 1. Meta task type

\begin{tabular}{ccccc}
\hline & $R$ & $P$ & $D$ & $A$ \\
\hline$R$ & $R \rightarrow R$ & $R \rightarrow P$ & $R \rightarrow D$ & $R \rightarrow A$ \\
$P$ & $P \rightarrow R$ & $P \rightarrow P$ & $P \rightarrow D$ & $P \rightarrow A$ \\
$D$ & $/$ & $D \rightarrow P$ & $D \rightarrow D$ & $D \rightarrow A$ \\
$A$ & $/$ & $/$ & $A \rightarrow D$ & $A \rightarrow A$ \\
\hline
\end{tabular}

The thirteen information flow relationships are defined as follows:

(1) $R \rightarrow R$ :It is an information sharing relationship. Two reconnaissance and early warning missions share information about their operation status and the detected targets within their respective responsibilities.

(2) $R \rightarrow P$ :It is an information transmission relationship. The reconnaissance and early warning mission provides intelligence information for the intelligence processing task, so that the intelligence processing task can process the intelligence information.

(3) $R \rightarrow D$ :It is an information transmission relationship. The reconnaissance and early warning mission provides intelligence information for the intelligence processing task, so that the intelligence processing task can process the intelligence information.

(4) $R \rightarrow A$ :It is an information transmission relationship. If the intelligence information obtained by the reconnaissance mission is of high quality, the response decision-making mission can directly use the information for combat operations.

(5) $P \rightarrow R$ :It is an order issue relationship, and the intelligence processing task can send relevant control instructions to the reconnaissance and early warning task according to its needs.

(6) $P \rightarrow P$ :It is an information sharing relationship. Different intelligence processing tasks can share their operation status, task load, comprehensive information generated, and target location status information to ensure the stability of their working status and the reliability of their results. 
(7) $P \rightarrow D$ :It is a kind of information transmission relationship, and the intelligence processing task transmits the integrated and integrated information it generates to the decision-making control task.

(8) $P \rightarrow A$ :It is an information transmission relationship. The intelligence processing task can send the high-precision intelligence information it generates to the response execution task to support its combat operations.

(9) $D \rightarrow P$ :It is an ordering relationship, and the decision-making control task can send relevant plans and requirements to the intelligence processing task according to its needs.

(10) $D \rightarrow D$ :It is an information sharing relationship. Different decision-making control tasks can exchange their operating conditions, task loads, combat plans and instructions, etc., to ensure their working status and ensure the reliability of the results.

(11) $D \rightarrow A$ :It is an ordering relationship, and the decision-making control task can send control commands and battle plans to the response execution unit.

(12) $A \rightarrow D$ :It is a kind of information transfer relationship, in response to the decision task, the current task status can be fed back to the decision control task.

(13) $A \rightarrow A$ :It is a kind of information sharing relationship, without responding to decision-making tasks, they can exchange their action status, location status information, etc., to ensure their working status and ensure the success rate of task execution.

\section{Case verification}

When a certain area encounters the threat of missile air strikes, the joint operations command headquarters will command the various services and units in various theaters to conduct joint anti-missile operations to protect the safety of our region's key areas. The main combat targets include various enemy air strike missiles.

\subsection{Combat scene setting}

According to the flow of anti-missile operations, the top-level mission tasks are divided into intelligence collection tasks, intelligence analysis tasks, command and control tasks, interception and strike tasks, protection tasks, and comprehensive support tasks. Then decompose each subtask in turn, and the final decomposition result is shown in the figure 5.

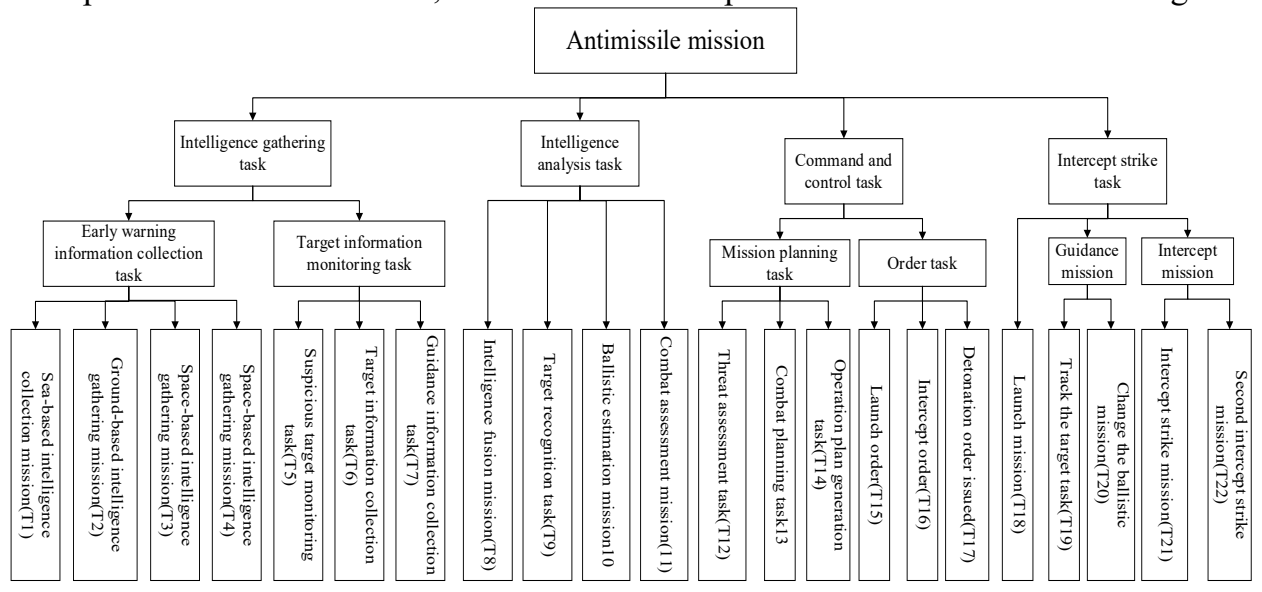

Fig. 5. Anti-missile mission breakdown. 
Suppose the mission task is $M$, after the task is decomposed, the set of meta tasks is obtained $\left\{T_{1}, T_{2}, T_{3}, \ldots T_{22}\right\}$, and then 22 meta tasks are modeled separately. Due to the limited space, the table shows the modeling results of some meta tasks.

Table 2. Meta task type.

\begin{tabular}{cc}
\hline Meta task sequence number & Type \\
\hline$T_{1} \sim T_{7}$ & $\mathrm{R}$ \\
$T_{8} \sim T_{11}$ & $\mathrm{P}$ \\
$T_{12} \sim T_{17}$ & $\mathrm{D}$ \\
$T_{18} \sim T_{22}$ & $\mathrm{~A}$ \\
\hline
\end{tabular}

Table 3. The nature of some P-type tasks.

\begin{tabular}{ccccccc}
\hline $\begin{array}{c}\text { Meta task } \\
\text { sequence number }\end{array}$ & Number & Location & Range & Datatype & Accuracy & Demand \\
\hline$T_{1}$ & 1 & $\left(x_{1}, y_{1}, z_{1}\right)$ & $r_{1}$ & image & 0.8 & $\bar{D}_{T 1}$ \\
$T_{2}$ & 2 & $\left(x_{2}, y_{2}, z_{2}\right)$ & $r_{2}$ & image & 0.8 & $\bar{D}_{T 2}$ \\
$\ldots$ & $\ldots$ & $\ldots$ & $\ldots$ & $\ldots$ & $\ldots$ & $\ldots$ \\
\hline
\end{tabular}

Table 4. The nature of some R-type tasks.

\begin{tabular}{ccccccccc}
\hline $\begin{array}{c}\text { Meta task } \\
\text { sequence } \\
\text { number }\end{array}$ & Number & Location & Range & Datatypes & Amount & Time & Demand \\
\hline$T_{8}$ & 8 & $\left(x_{8}, y_{8}, z_{8}\right)$ & $r_{8}$ & $\begin{array}{c}\text { Image } \\
\text { signal } \\
\ldots\end{array}$ & 0.8 & $t_{1}$ & $\bar{D}_{T 8}$ \\
$T_{9}$ & 9 & $\left(x_{9}, y_{9}, z_{9}\right)$ & $r_{9}$ & $\begin{array}{c}\text { image } \\
\text { signal } \\
\ldots\end{array}$ & 0.8 & $t_{2}$ & $\bar{D}_{T 9}$ \\
$\ldots$ & $\ldots$ & $\ldots$ & $\ldots$ & $\ldots$ & $\ldots$ & $\ldots$ & $\ldots$ \\
\hline
\end{tabular}

Table 5. The nature of some D-type tasks.

\begin{tabular}{cccccccc}
\hline $\begin{array}{c}\text { Meta task } \\
\text { sequence } \\
\text { number }\end{array}$ & Number & Location & Range & Amount & Time & Level & Demand \\
\hline$T_{12}$ & 12 & $\left(x_{12}, y_{12}, z_{12}\right)$ & $r_{12}$ & 0.8 & $t_{1}$ & tactics & $\bar{D}_{T 12}$ \\
$T_{13}$ & 13 & $\left(x_{13}, y_{13}, z_{13}\right)$ & $r_{13}$ & 0.8 & $t_{2}$ & tactics & $\bar{D}_{T 13}$ \\
$\ldots$ & $\ldots$ & $\ldots$ & $\ldots$ & $\ldots$ & $\ldots$ & $\ldots$ & $\ldots$ \\
\hline
\end{tabular}

Table 6. The nature of some A-type tasks.

\begin{tabular}{ccccc}
\hline Meta task sequence number & Number & Location & Range & Demand \\
\hline$T_{18}$ & 18 & $\left(x_{18}, y_{18}, z_{18}\right)$ & $r_{18}$ & $\bar{D}_{T 18}$ \\
$T_{19}$ & 19 & $\left(x_{19}, y_{19}, z_{19}\right)$ & $r_{19}$ & $\bar{D}_{T 19}$
\end{tabular}

In this case, the operational capabilities involved are set as follows: 1. Early warning capability 2 . Reconnaissance and guidance capability 3. Data processing capability 4. Data 
analysis capability 5. Operational command and management capability 6. Operational control capability 7. Communication capability 8 . Target search and tracking capability 9. Interception capability 10. Battlefield position defense capability 11. Support capability. Respectively expressed as $A=\left\{A_{1}, A_{2}, A_{3} \ldots A_{11}\right\}$, where the specific value represents the per unit value of the quantity of resources.

demand vector $\bar{D}_{T n}$ can be expressed as:

Table 7. Demand vector D.

\begin{tabular}{ccccccccccccc}
\hline $\begin{array}{c}\text { Meta } \\
\text { task } \\
\text { sequence } \\
\text { number }\end{array}$ & Demand & $A_{1}$ & $A_{2}$ & $A_{3}$ & $A_{4}$ & $A_{5}$ & $A_{6}$ & $A_{7}$ & $A_{8}$ & $A_{9}$ & $A_{10}$ & $A_{11}$ \\
\hline$T_{1}$ & $\bar{D}_{T 1}$ & 3 & 0 & 0 & 0 & 0 & 0 & 0 & 0 & 0 & 0 & 0 \\
$T_{2}$ & $\bar{D}_{T 2}$ & 4 & 4 & 0 & 0 & 0 & 0 & 0 & 0 & 0 & 0 & 0 \\
$T_{3}$ & $\bar{D}_{T 3}$ & 4 & 0 & 0 & 0 & 0 & 0 & 0 & 0 & 0 & 0 & 0 \\
$T_{4}$ & $\bar{D}_{T 4}$ & 6 & 6 & 0 & 0 & 0 & 0 & 0 & 2 & 0 & 0 & 0 \\
$T_{5}$ & $\bar{D}_{T 5}$ & 3 & 3 & 0 & 0 & 0 & 0 & 0 & 5 & 0 & 0 & 0 \\
$\cdots$ & $\cdots$ & $\cdots$ & $\cdots$ & $\cdots$ & $\cdots$ & $\cdots$ & $\cdots$ & $\cdots$ & $\cdots$ & $\cdots$ & $\cdots$ & $\cdots$ \\
\hline
\end{tabular}

\subsection{Meta task relationship construction}

Analyze the execution sequence of the 22 subtasks, and determine the relationship between the tasks according to their occurrence. The relationship results are shown in the figure 6.Refer to the table for the comparison of numbers and relationships in the table 8 .

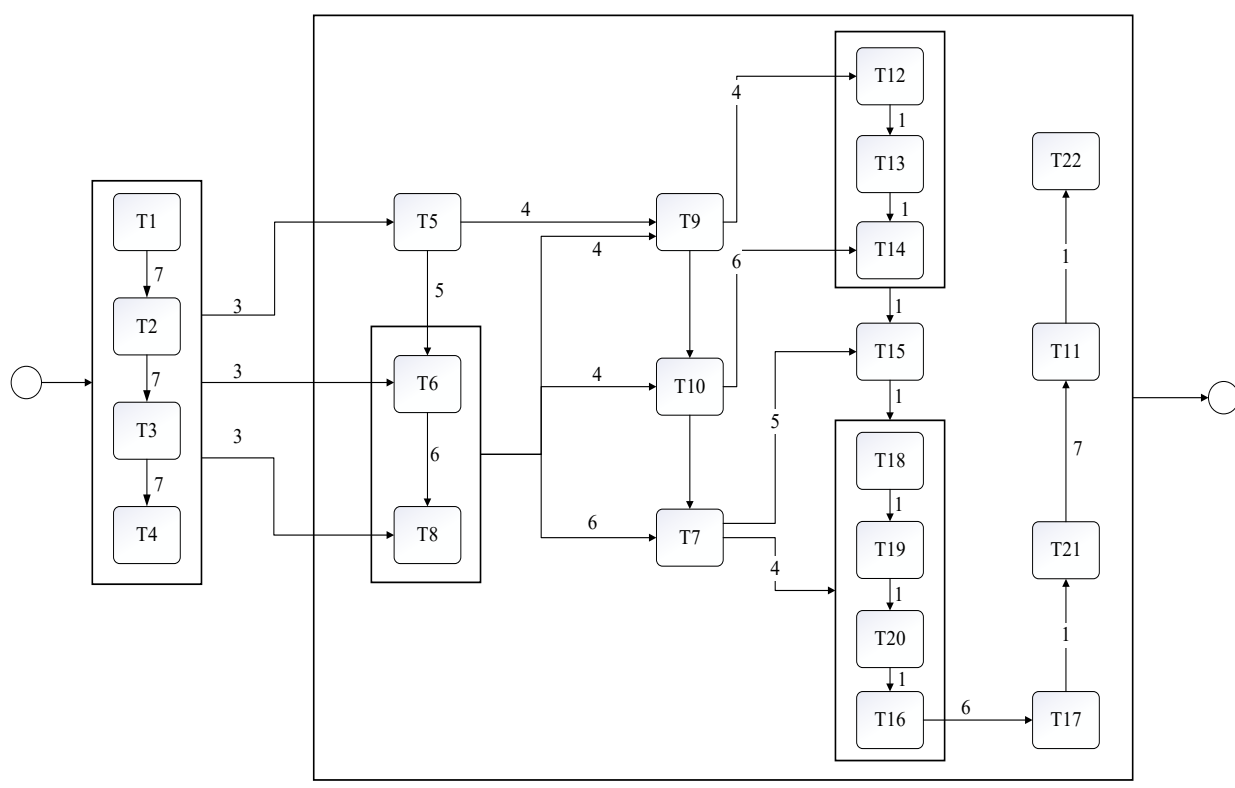

Fig. 6. Time relationships. 
Table 8. Numbering relationship comparison chart.

\begin{tabular}{ccc}
\hline Number & Relation & Relational expression \\
\hline 1 & Serial meets & $\operatorname{Serial}_{\text {meets }}\left(T_{i}, T_{j}\right)$ \\
2 & Serial before & $\operatorname{Serial}_{\text {before }}\left(T_{i}, T_{j}\right)$ \\
3 & Serial overlaps & $\operatorname{Serial}_{\text {overlaps }}\left(T_{i}, T_{j}\right)$ \\
4 & Parallel during & parallel $_{\text {during }}\left(T_{i}, T_{j}\right)$ \\
5 & Parallel starts & parallel $_{\text {starts }}\left(T_{i}, T_{j}\right)$ \\
6 & Parallel finishes & parallel $_{\text {finishes }}\left(T_{i}, T_{j}\right)$ \\
7 & Parallel equal & parallel $_{\text {equal }}\left(T_{i}, T_{j}\right)$
\end{tabular}

Define the information flow relationship between meta tasks according to the previous definition, as shown in the table 9.

Finally, the information relationship is shown on the time relationship diagram, as shown in figure. The dotted lines in the figure 7 represent information relationships, and the numbers on the connecting lines represent different types of relationships, which have been indicated in the above table 9.

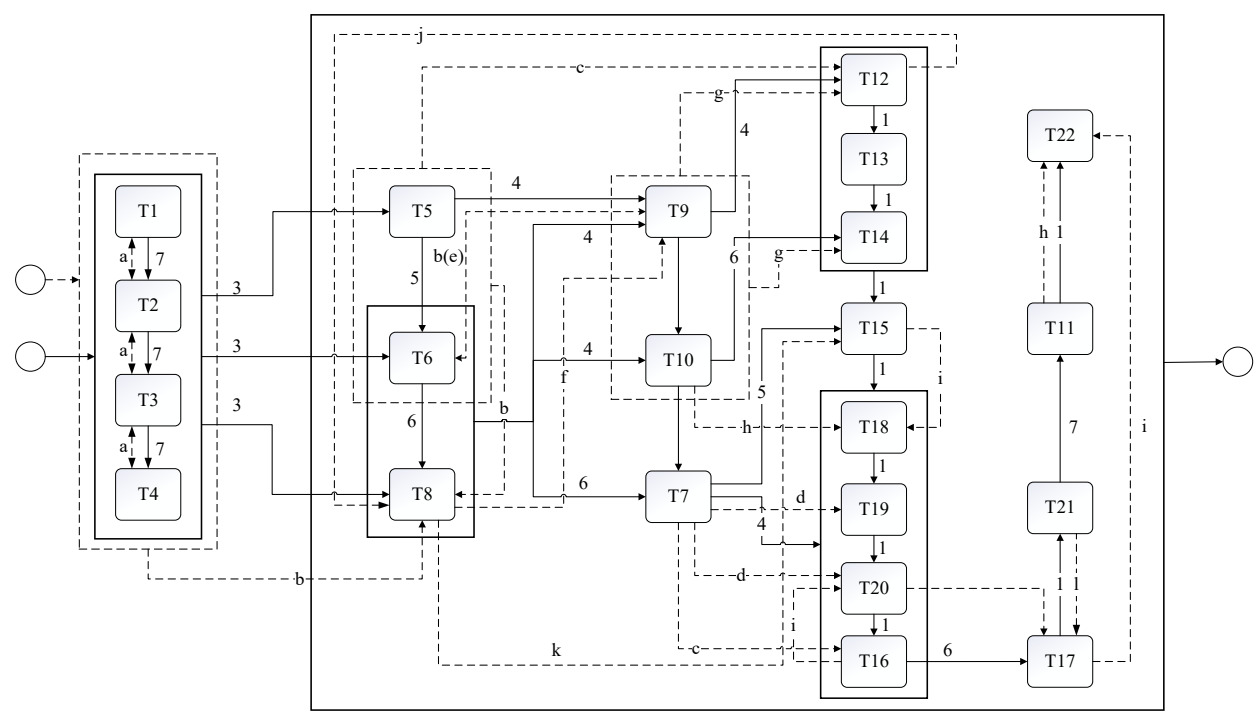

Fig. 7. Information relationships.

\section{Conclusion}

This paper focuses on solving two problems and has carried out three parts of work: (1) The mission task is decomposed, and high-level mission tasks are decomposed level by level, and they are transformed into meta tasks that can be directly understood by the combat unit, and According to the OODA combat theory, the reconnaissance and early warning tasks, intelligence processing tasks, decision-making control tasks, and response execution tasks are defined and formalized descriptions are made. (2) Constructed the relationship between meta tasks. From the perspectives of time and information, respectively constructed the 
relationship between meta tasks. (3) According to the theoretical methods proposed above, case studies were conducted to prove the theory The feasibility of the method.

Table 9. Meta task information flow relationship.

\begin{tabular}{|c|c|c|c|}
\hline Number & Type & Meta task & Describe \\
\hline a & $R \rightarrow R$ & $T_{1} \leftrightarrow T_{2} \leftrightarrow T_{3} \leftrightarrow T_{4}$ & $\begin{array}{l}\text { Sea-based, ground-based, air-based, and } \\
\text { space-based intelligence sharing }\end{array}$ \\
\hline \multirow{3}{*}{$\mathrm{b}$} & \multirow{3}{*}{$R \rightarrow P$} & $T_{1}, T_{2}, T_{3}, T_{4} \rightarrow T_{8}$ & $\begin{array}{l}\text { Intelligence collection mission supports } \\
\text { intelligence fusion mission }\end{array}$ \\
\hline & & $T_{5}, T_{6} \rightarrow T_{8}$ & $\begin{array}{l}\text { The information collected about the target } \\
\text { is handed over to the intelligence fusion } \\
\text { task }\end{array}$ \\
\hline & & $T_{6} \rightarrow T_{9}$ & $\begin{array}{l}\text { Pass the target information to the target } \\
\text { recognition task for recognition }\end{array}$ \\
\hline \multirow{2}{*}{$\mathrm{c}$} & \multirow{2}{*}{$R \rightarrow D$} & $T_{5}, T_{6} \rightarrow T_{12}$ & $\begin{array}{l}\text { Perform threat assessment based on the } \\
\text { target information obtained by the } \\
\text { reconnaissance platform }\end{array}$ \\
\hline & & $T_{7} \rightarrow T_{16}$ & $\begin{array}{l}\text { Based on guidance information to issue } \\
\text { interception commands for intercepting } \\
\text { missiles }\end{array}$ \\
\hline d & $R \rightarrow A$ & $T_{7} \rightarrow T_{19}, T_{20}$ & $\begin{array}{l}\text { The interceptor missile adjusts its } \\
\text { trajectory directly through guidance } \\
\text { information }\end{array}$ \\
\hline $\mathrm{e}$ & $P \rightarrow R$ & $T_{9} \rightarrow T_{6}$ & $\begin{array}{l}\text { Target recognition task adjusts the } \\
\text { collection of target information as needed }\end{array}$ \\
\hline $\mathrm{f}$ & $P \rightarrow P$ & $T_{8} \leftrightarrow T_{9}$ & $\begin{array}{l}\text { Cooperation between intelligence fusion } \\
\text { task and target recognition task }\end{array}$ \\
\hline \multirow[t]{2}{*}{ g } & \multirow{2}{*}{$P \rightarrow D$} & $T_{9}, T_{10} \rightarrow T_{12}$ & $\begin{array}{l}\text { Perform threat assessment based on the } \\
\text { identified target category and estimated } \\
\text { trajectory }\end{array}$ \\
\hline & & $T_{9}, T_{10} \rightarrow T_{14}$ & $\begin{array}{l}\text { Design combat plans based on target } \\
\text { categories and estimated ballistics }\end{array}$ \\
\hline \multirow{2}{*}{$\mathrm{h}$} & \multirow{2}{*}{$P \rightarrow A$} & $T_{10} \rightarrow T_{18}$ & $\begin{array}{l}\text { Calculate the launch parameters of the } \\
\text { interceptor missile through the estimated } \\
\text { trajectory }\end{array}$ \\
\hline & & $T_{11} \rightarrow T_{22}$ & $\begin{array}{l}\text { Determine whether a second interception } \\
\text { is required based on the results of the } \\
\text { strike assessment }\end{array}$ \\
\hline \multirow{3}{*}{$\mathrm{i}$} & \multirow{3}{*}{$D \rightarrow A$} & $T_{15} \rightarrow T_{18}$ & $\begin{array}{c}\text { Give instructions to the interceptor } \\
\text { missile launch }\end{array}$ \\
\hline & & $T_{16} \rightarrow T_{20}$ & $\begin{array}{c}\text { Change the trajectory of the interceptor } \\
\text { missile to intercept and issue an } \\
\text { instruction }\end{array}$ \\
\hline & & $T_{17} \rightarrow T_{21}, T_{22}$ & $\begin{array}{l}\text { Give instructions to intercepting missiles } \\
\text { for one or two strikes }\end{array}$ \\
\hline $\mathrm{j}$ & $D \rightarrow P$ & $T_{12} \rightarrow T_{8}$ & $\begin{array}{c}\text { Threat assessment requires } \\
\text { comprehensive intelligence for real-time } \\
\text { updates }\end{array}$ \\
\hline $\mathrm{k}$ & $D \rightarrow D$ & $T_{8} \leftrightarrow T_{15}$ & $\begin{array}{l}\text { The launch timing needs to be selected in } \\
\text { a timely manner based on the degree of } \\
\text { threat assessment }\end{array}$ \\
\hline 1 & $A \rightarrow D$ & $T_{21} \rightarrow T_{17}$ & $\begin{array}{c}\text { Choose the timing of explosion according } \\
\text { to the information of changing the } \\
\text { ballistic mission }\end{array}$ \\
\hline
\end{tabular}


Equipment advance research: space XXXX demand generation technology research, No. 30504010104

\section{References}

1. Yang Shixing and Yang Dongsheng 2009 Combat mission decomposition and mission modeling method on Firepower and Command Control vol 08 pp 24-29.

2. Wang Wei Liu Fuxian. 2017 Combat task decomposition optimization based on coordination correlation on Firepower and Command Control vol 11 pp 107-110.

3. Yan Jingjing and Xu Ying. 2018 Research on the Application of Conceptual Model of Maritime Area Air Defense Mission. China Command and Control Society. (eds.) Proceedings of the Sixth China Command and Control Conference Vol 2 pp.272-277.

4. Dong Tao, Liu Fuxian \& Li Xiang. 2012. Task decomposition of the final stage of multi-layer anti-missile warfare on Modern Defense Technology vol 04, pp 17-20.

5. Boyd, J. R. 1987. A Discourse on Winning and Losing.

6. Allen, J. F. 1990 Maintaining knowledge about temporal intervals. Readings in Qualitative Reasoning About Physical Systems 26(11) pp 361-372. 